\title{
Simultaneous spatial and temporal focusing for tissue ablation
}

\author{
Erica Block, ${ }^{1}$ Michael Greco, ${ }^{1}$ Dawn Vitek, ${ }^{1}$ Omid Masihzadeh, ${ }^{2}$ David A. Ammar, ${ }^{2}$ \\ Malik Y. Kahook, ${ }^{2}$ Naresh Mandava, ${ }^{2}$ Charles Durfee, ${ }^{1}$ and Jeff Squier ${ }^{1, *}$ \\ ${ }^{1}$ Department of Physics, Colorado School of Mines, 1523 Illinois Street, Golden, CO 80401, USA \\ ${ }^{2}$ Department of Ophthalmology, University of Colorado School of Medicine, Aurora CO, 80045, USA \\ *jsquier@mines.edu
}

\begin{abstract}
Simultaneous spatial temporal focusing (SSTF) is used to deliver microjoule femtosecond pulses with low numerical aperture geometries $(<0.05$ NA) with characteristics that are significantly improved compared to standard focusing paradigms. Nonlinear effects that would normally result in focal plane shifts and focal spot distortion are mitigated when SSTF is employed. As a result, it is shown that SSTF will enable surgical implementations that are presently inhibited.
\end{abstract}

(C)2013 Optical Society of America

OCIS codes: (140.3390) Laser materials processing; (190.4360) Nonlinear optics, devices.

\section{References and links}

1. P. Pronko, S. Dutta, J. Squier, V. Rudd, D. Du, and G. Mourou, "Machining of submicron holes using a femtosecond laser at $800 \mathrm{~nm}$," Opt. Commun. 114(1-2), 106-110 (1995).

2. C. B. Schaffer, A. Brodeur, J. F. García, and E. Mazur, "Micromachining bulk glass by use of femtosecond laser pulses with nanojoule energy," Opt. Lett. 26(2), 93-95 (2001).

3. T. Juhasz, G. A. Kastis, C. Suárez, Z. Bor, and W. E. Bron, "Time-resolved observations of shock waves and cavitation bubbles generated by femtosecond laser pulses in corneal tissue and water," Lasers Surg. Med. 19(1), 23-31 (1996).

4. T. Juhasz, F. H. Loesel, R. M. Kurtz, C. Horvath, J. F. Bille, and G. Mourou, "Corneal refractive surgery with femtosecond lasers,” IEEE J. Sel. Top. Quantum Electron. 5(4), 902-910 (1999).

5. R. M. Kurtz, C. Horvath, H. H. Liu, R. R. Krueger, and T. Juhasz, "Lamellar refractive surgery with scanned intrastromal picosecond and femtosecond laser pulses in animal eyes," J. Refract. Surg. 14(5), 541-548 (1998).

6. D. Oron, E. Tal, and Y. Silberberg, "Scanningless depth-resolved microscopy," Opt. Express 13(5), 1468-1476 (2005).

7. G. Zhu, J. van Howe, M. Durst, W. Zipfel, and C. Xu, "Simultaneous spatial and temporal focusing of femtosecond pulses," Opt. Express 13(6), 2153-2159 (2005).

8. F. He, H. Xu, Y. Cheng, J. Ni, H. Xiong, Z. Xu, K. Sugioka, and K. Midorikawa, "Fabrication of microfluidic channels with a circular cross section using spatiotemporally focused femtosecond laser pulses," Opt. Lett. 35(7), 1106-1108 (2010).

9. D. N. Vitek, D. E. Adams, A. Johnson, P. S. Tsai, S. Backus, C. G. Durfee, D. Kleinfeld, and J. A. Squier, "Temporally focused femtosecond laser pulses for low numerical aperture micromachining through optically transparent materials," Opt. Express 18(17), 18086-18094 (2010).

10. D. N. Vitek, E. Block, Y. Bellouard, D. E. Adams, S. Backus, D. Kleinfeld, C. G. Durfee, and J. A. Squier, "Spatio-temporally focused femtosecond laser pulses for nonreciprocal writing in optically transparent materials," Opt. Express 18(24), 24673-24678 (2010).

11. R. Kammel, R. Ackermann, A. Tünnermann, and S. Nolte, "Pump-probe investigation of fs-LIOB in water by simultaneous spatial and temporal focusing," Proc. SPIE 8611, 86110A, 86110A-7 (2013).

12. C. G. Durfee, M. Greco, E. Block, D. Vitek, and J. A. Squier, "Intuitive analysis of space-time focusing with double-ABCD calculation," Opt. Express 20(13), 14244-14259 (2012).

13. J. H. Marburger, "Self-focusing: theory," Prog. Quantum Electron. 4, 35-110 (1975).

14. D. Giguère, G. Olivié, F. Vidal, S. Toetsch, G. Girard, T. Ozaki, J.-C. Kieffer, O. Nada, and I. Brunette, "Laser ablation threshold dependence on pulse duration for fused silica and corneal tissues: experiments and modeling," J. Opt. Soc. Am. A 24(6), 1562-1568 (2007).

15. L. Hoffart, P. Lassonde, F. Légaré, F. Vidal, N. Sanner, O. Utéza, M. Sentis, J. C. Kieffer, and I. Brunette, "Surface ablation of corneal stroma with few-cycle laser pulses at $800 \mathrm{~nm}$," Opt. Express 19(1), 230-240 (2011).

\section{Introduction}

The innovation that resulted in the application of femtosecond lasers to micromachining $[1,2]$ and ophthalmic surgeries [3-5] was the realization that the damage process went from a 
statistical to deterministic phenomenon with a well-defined threshold when the pulse duration was scaled from the nanosecond to the femtosecond regime. This has enabled precise surface ablation of a broad range of materials and biological tissues. Despite this precision, in terms of surgical applications there are several key aspects of these systems that confound the potential of femtosecond laser-based tissue ablation. These include the variation in tissue resulting in a disparity of ablation thresholds, naturally occurring apertures (that limit the numerical aperture [NA] and working distance of the delivery beam), and the location of sensitive membranes that can be within micrometers of the targeted structure(s). The combination of these last two parameters results in a seemingly intractable conundrum, and become further complicated by the real-world realization that pulsed lasers exhibit shot-to-shot energy variation. At low NA and at depth within a tissue, nonlinear effects, such as self-focusing, result in focal plane jitter that make precise cutting adjacent to sensitive boundaries difficult.

We demonstrate that these issues that appear intractable can be addressed with simultaneous spatio-temporal focusing (SSTF) [6,7]. In a SSTF system, the excitation beam is spectrally dispersed into a collimated series of low NA beamlets prior to the focusing optic. After the focusing optic, the beams are angularly dispersed, only intersecting at the focal plane in a single uniform diffraction-limited spot in space, and transform-limited pulse in time. The NA of the beamlets determines the final focal spot size. As a result of the lack of complete spectral overlap in the out-of-focus regions, the peak power and correlating intensity of the beam is markedly lower in these zones. The reduced out-of-focus intensity mitigates integrated nonlinear effects such as self-focusing. The utility of the SSTF focal geometry for micromachining, was first demonstrated by $\mathrm{He}$ et al [8] with application to microfluidics, and Vitek et al $[9,10]$ with application to low numerical aperture machining at millimeter depths inside optically transparent materials. Vitek et al showed that targeted ablation with $50 \mu \mathrm{J}, 60$ fs pulses at $0.05 \mathrm{NA}$ through $6 \mathrm{~mm}$ of fused silica was possible with SSTF, while these same beam parameters resulted in filamentation and tracking throughout the solid when a standard focal geometry was used. Finally, Kammel et al [11] have recently shown that SSTF improves the localization of breakdown in water.

In this paper, we extend this work to include biological tissue, with measurements that explicitly demonstrate registration of the focal plane in SSTF. This is compared with a standard focusing geometry where the focal plane shifts and the focus distorts as a function of the pulse energy. Further, we demonstrate that the extension of the ablation zone is axially restricted despite cutting 4 to 58 times above the ablation threshold. This directly addresses key aspects required for a more intelligent surgical implementation of femtosecond laser pulses. These are (1) the ability to cut with a broad range of fluences without impacting the location of the focal plane, which is desirable given tissue differences (2) the use of beams which have longer working distances facilitating delivery, and (3) precise axial ablation with these beams enabling safer penetration up to sensitive boundaries or membranes.

\#185757 - \$15.00 USD Received 21 Feb 2013; revised 11 Apr 2013; accepted 6 May 2013; published 8 May 2013

(C) 2013 OSA 1 June 2013 | Vol. 4, No. 6 | DOI:10.1364/BOE.4.000831 | BIOMEDICAL OPTICS EXPRESS 832 


\section{Impact of the beam aspect ratio on axial confinement}

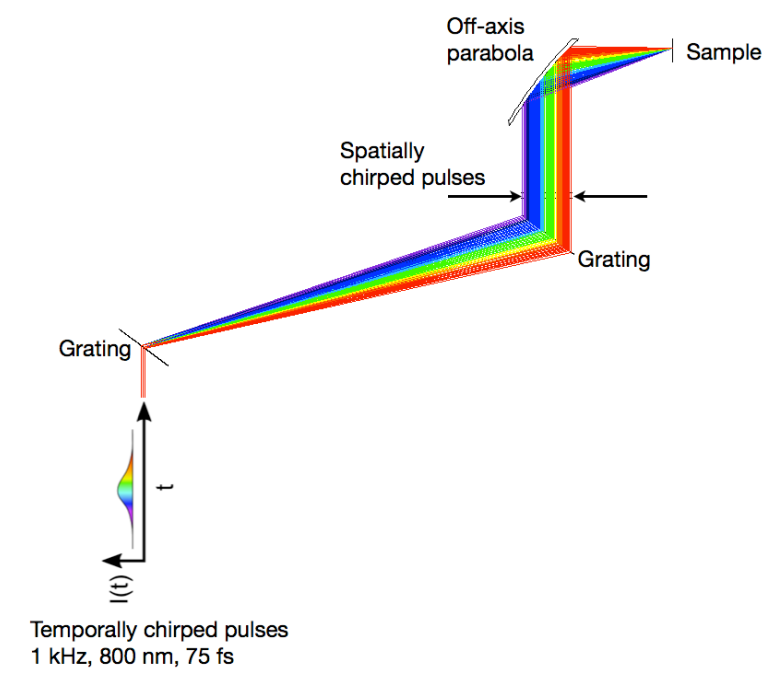

Fig. 1. The simultaneous spatial temporal focusing geometry.

The optical arrangement for SSTF must produce a beam with a transverse spatial chirp (collimated frequency components) along with collimated beamlets. In most of our experiments, we employ the SSTF geometry described in $[9,10]$, shown in Fig. 1. A positively chirped pulse from a $75 \mathrm{fs}, 1 \mathrm{kHz}$, chirped pulse amplification (CPA) Ti:sapphire system is directed through a $600 \mathrm{l} / \mathrm{mm}$ grating pair (Thorlabs, \#GR25-0608) arranged in single pass configuration. The collimated, spatially chirped output is typically focused by a $25.4 \mathrm{~mm}$ focal length, $25.4 \mathrm{~mm}$ clear aperture 90-degree off-axis parabola (Janos Technology, \#A8037175). An important parameter with SSTF is the beam aspect ratio, $\beta_{B A}$ [12]. This is simply the ratio of the width of the collimated spatially chirped beam (output of the grating pair) relative to the beam diameter at the input to the grating pair. Figure 2 is an illustration of the dramatic reduction in the axial beam intensity for an SSTF focusing geometry. In this instance a focal spot of $33 \mu \mathrm{m}$ (measured $1 / \mathrm{e}^{2}$ diameter of the central intensity) is used in both cases. Without SSTF the axial intensity full width at half maximum (FWHM) is $8.6 \mathrm{~mm}$ as shown by the black intensity contour in Fig. 2(b). This stands in marked contrast to the SSTF beam, where the FWHM is reduced by a factor of 59 to $146 \mu \mathrm{m}$ (red intensity contour Fig. 2(b)). The $\beta_{B A}$ for these simulations was 11 . 

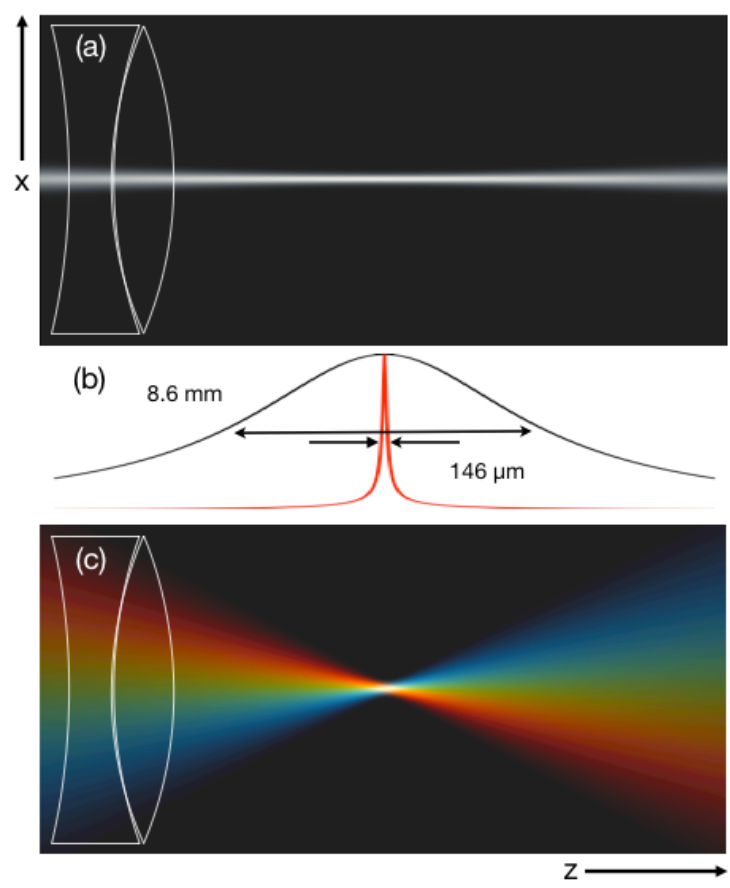

Fig. 2. (a) Intensity profile of a beam focused to a $33 \mu \mathrm{m}$ spot size (1/ $\mathrm{e}^{2}$ beam diameter) using a standard focal geometry. (b) Integrated intensity contours along the axial dimension of the focus without SSTF (black) and with SSTF (red). (c) Intensity profile of a beam with an SSTF focusing geometry also focused to a $33 \mu \mathrm{m}$ spot size. The beam aspect ratio, $\beta_{B A}$, is 11 .

The reduction in the axial intensity profile of the SSTF beam is governed by $\beta_{B A}$ [12]. Beam aspect ratios $>10$ are readily achieved for microjoule pulses using standard 1" diameter focusing optics. As a useful rule of thumb, a $\beta_{B A} \sim 4$ results in an order of magnitude decrease in the axial intensity FWHM. Explicitly, the intensity scales with $\beta_{B A}$ according to the expression

$$
I(z)=I_{0} \frac{1}{\sqrt{\left(1+\frac{z^{2}}{z_{R}^{2}} \beta_{B A}^{4}\right)\left(1+\frac{z^{2}}{z_{R}^{2}}\right)}}
$$

where $\mathrm{I}_{0}$ represents the peak intensity at the focal plane and $\mathrm{z}_{\mathrm{R}}$ is the Rayleigh range of the non-SSTF beam [12]. Given the radical reduction in the intensity outside of the focal plane with the increase in $\beta_{B A}$, integral nonlinear effects that are accumulated as the pulse propagates to the target focal position are minimized. For example, the nonlinear phase accumulation represented by the B-integral is calculated to be reduced by a factor of 10 for a $\beta_{B A} \sim 6$. Consequently, SSTF geometries mitigate effects such as self-focusing. This is a legitimate concern when dealing with microjoule, low numerical aperture beams, for example, since the peak powers for these beam conditions often exceed the critical power for selffocusing, especially when employing pulse energies well above the threshold for damage.

We performed an experiment in which energetic pulses were focused in air to explicitly demonstrate the resistance of SSTF pulses to self-focusing and ionization-induced defocusing. A 45-degree off-axis parabola (Edmund Optics \#83-970), $\mathrm{f}=76.2 \mathrm{~mm}$, was used to focus 40 fs pulses at $800 \mathrm{~nm}$ from a $1 \mathrm{kHz}$ chirped pulse amplification system in air to a $8 \mu \mathrm{m}$ spot size 
(FWHM) using both SSTF and conventional (non-SSTF) focusing geometries. The plasma breakdown was imaged from above and recorded as a function of increasing pulse energy. Figure 3 shows the focal spot intensity profile for a focal geometry without SSTF (left panels). At $104 \mu \mathrm{J}$ the breakdown is axially symmetric and centered on the focal plane (white dashed line). When the energy is increased to $190 \mu \mathrm{J}$ the focus has shifted $60 \mu \mathrm{m}$ toward the parabola, and remains reasonably symmetric. At $278 \mu \mathrm{J}$, the focal spot shifts by $130 \mu \mathrm{m}$ and becomes distorted. By the time the energy reaches $358 \mu \mathrm{J}$ (maximum throughput for the double-pass, conventional compressor), the distorted profile shifts $160 \mu \mathrm{m}$ away from the focal plane. This is classic self-focusing behavior as predicted by Marburger [13]. Further increasing the pulse energies results in beam filamentation.
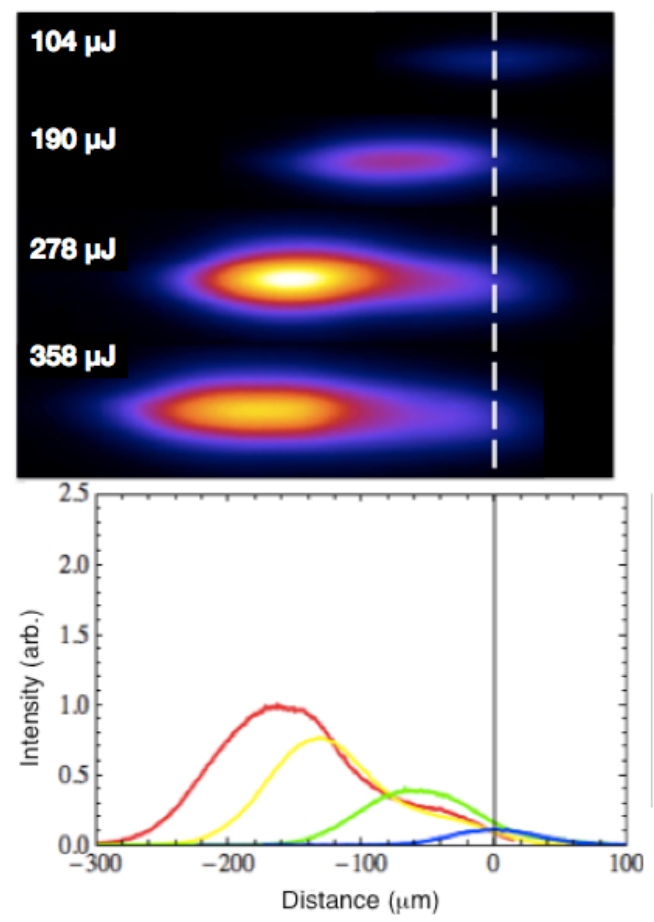
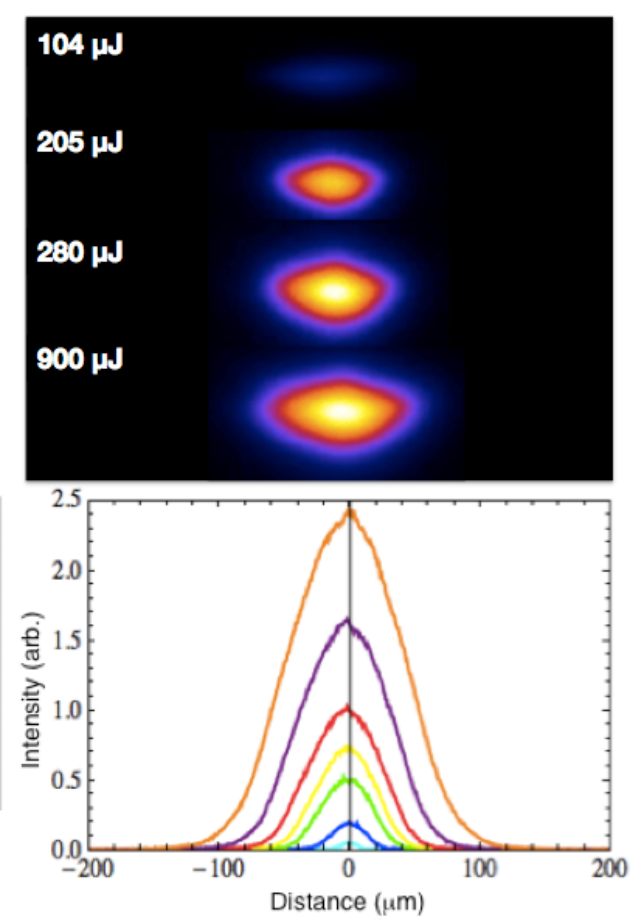

Fig. 3. Image of focus as a function of pulse energy for a standard focal geometry (left) and an SSTF focal geometry (right). The dashed line in the left panel indicates the focal plane of the focusing optic. The beam's focal position shifts toward this focusing optic as the energy is increased.

By comparison, the axial position of the focus does not shift when SSTF is introduced (Fig. 3, right panels, $\beta_{B A}=5.4$ ). Beginning at the lowest pulse energy value of $104 \mu \mathrm{J}$, the focusing behavior is similar for both focusing geometries. Then, as the energy is increased, with SSTF there is no visible shift in the profile position or shape. Even at $900 \mu \mathrm{J}$ (maximum throughput for the single pass SSTF compressor) the profile remains centered and undistorted. At this point, the pulse has effectively an order of magnitude more energy than the maximum pulse energy that could be used with a standard focus $(104 \mu \mathrm{J})$ and still maintains a symmetric profile centered on the focal plane.

This behavior has important implications in surgical implementations. First, pulse-to-pulse energy fluctuations will have negligible impact on the location and shape of the focus in an SSTF system. Second, the cutting fluence can be increased substantially above threshold without affecting the focal position making it possible to handle a broader range of tissue conditions. 


\section{Tissue ablation above the ablation threshold}

The morphology of laser ablation in tissue was investigated for focusing geometries with and without SSTF. For both focal geometries laser parameters, aside from $\beta_{B A}$, are kept identical. The beam is focused on the surface of the lenses of porcine eyes such that the peak fluence is $5.2 \mathrm{~J} / \mathrm{cm}^{2}$ which is $\sim 4$ times above the ablation threshold $[14,15]$. The pulse repetition rate is 1 $\mathrm{kHz}$; the central wavelength is $800 \mathrm{~nm}$; and the pulse duration is $75 \mathrm{fs}$.

First, a standard focusing geometry is used. The beam is focused on the surface of the porcine lens such that it is normally incident with the sample, having a $33 \mu \mathrm{m}$ spot size $\left(1 / \mathrm{e}^{2}\right.$ diameter) with a 90 -degree off-axis parabola, $f=25 \mathrm{~mm}$. The sample is translated laterally at a rate of $0.6 \mathrm{~mm} / \mathrm{s}(\sim 55$ pulses per $33 \mu \mathrm{m}$ spot $)$. Multiple passes were made such that 4 distinct lines (in rows, separated by $200 \mu \mathrm{m}$ ) are cut across the full aperture of the porcine lens. Figure 4 is a single-frame excerpt from a video recording (Media 1) that shows the cutting process live under these conditions.

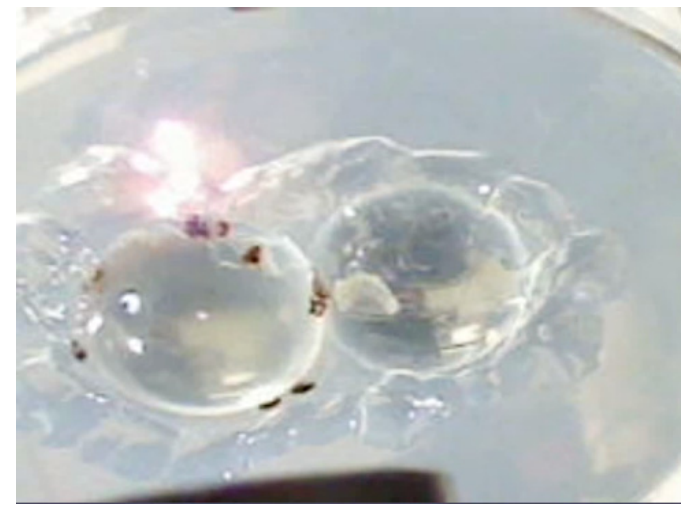

Fig. 4. Single-frame excerpt from a video recording of laser ablation on the surface of a porcine lens mounted in agarose using a standard focusing geometry (Media 1, $685 \mathrm{~KB})$. Two lenses are shown; however, only the lens on the left is being ablated.

Notably, without SSTF, nonlinear effects such as supercontinuum generation are visible with the focus positioned as far as $1 \mathrm{~cm}$ above the lens, and grow more intense when the focal spot is positioned at the lens' surface. The first pass across the lens results in the lens tissue opening in the form of a large welt, followed by the propagation of a bubble that grows to encompass a significant portion of the lens' surface. A montage of the bubble propagation is detailed in Fig. 5 to more clearly delineate the propagation as the cut progresses. Finally, we note that at these beam sizes and fluences even the out-of-focus beam would periodically damage the system's optics such as the turning mirrors. 


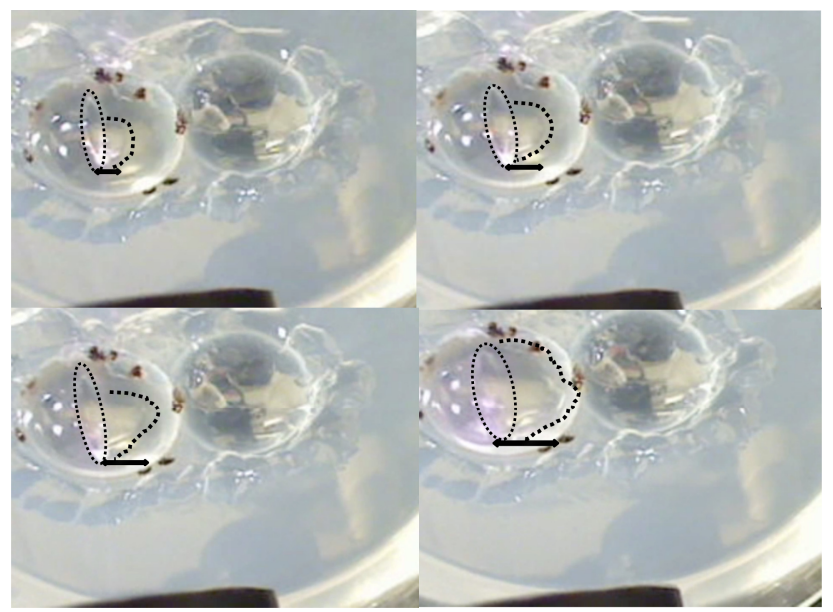

Fig. 5. Montage that details the propagation of the bubbles that follow the first cut with the standard focusing geometry.

The quality of the cut improves with SSTF. Figure 6 is a single-frame excerpt from a video recording (Media 2) using the SSTF focusing geometry under otherwise identical conditions, including the fluence, spot size, translation speed and raster pattern. The beam aspect ratio is $\beta_{B A}=11$. Note that no welt forms, and no large bubbles are generated. (Microscopic bubble formation, which can pose a problem in these surgeries, was not investigated.) In addition, when the focal plane is $1 \mathrm{~cm}$ above the lens, there is no visible supercontinuum generation and no damage to the optics.

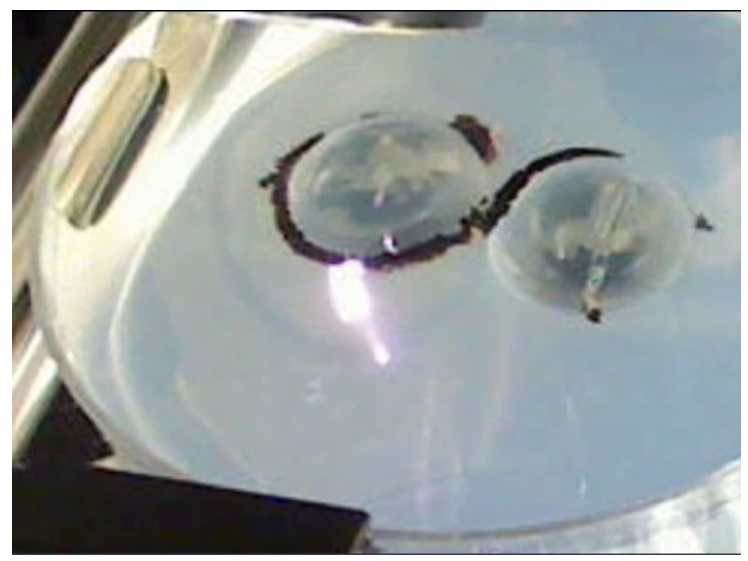

Fig. 6. Single-frame excerpt from a video recording of channels cut across the surface of a porcine lens mounted in agarose, using a SSTF focusing geometry (Media 2, $731 \mathrm{~KB}$ ).

Laser ablated tissues from both standard and SSTF focusing geometries were examined histologically. Lenses were prepared according to the following procedure: They were preserved overnight at $4{ }^{\circ} \mathrm{C}$ in Fekete's fixative ( $4 \%$ formaldehyde, $5 \%$ glacial acetic acide, $70 \%$ ethanol) and then embedded in paraffin. Histological sections $(8 \mu \mathrm{m}$ thick $)$ were stained with Mayer's hematoxylin and eosin Y (H\&E: Richard-Allan Scientific, Kalamazoo, MI). Bright field imaging was performed using a Nikon Eclipse 80i microscope (Nikon, Melville, NY) equipped with a Nikon D5-Fil color camera and a Nikon 4x/Plan Fluor objective lens. Multiple representative images of the tissue histology are shown in Fig. 7. 

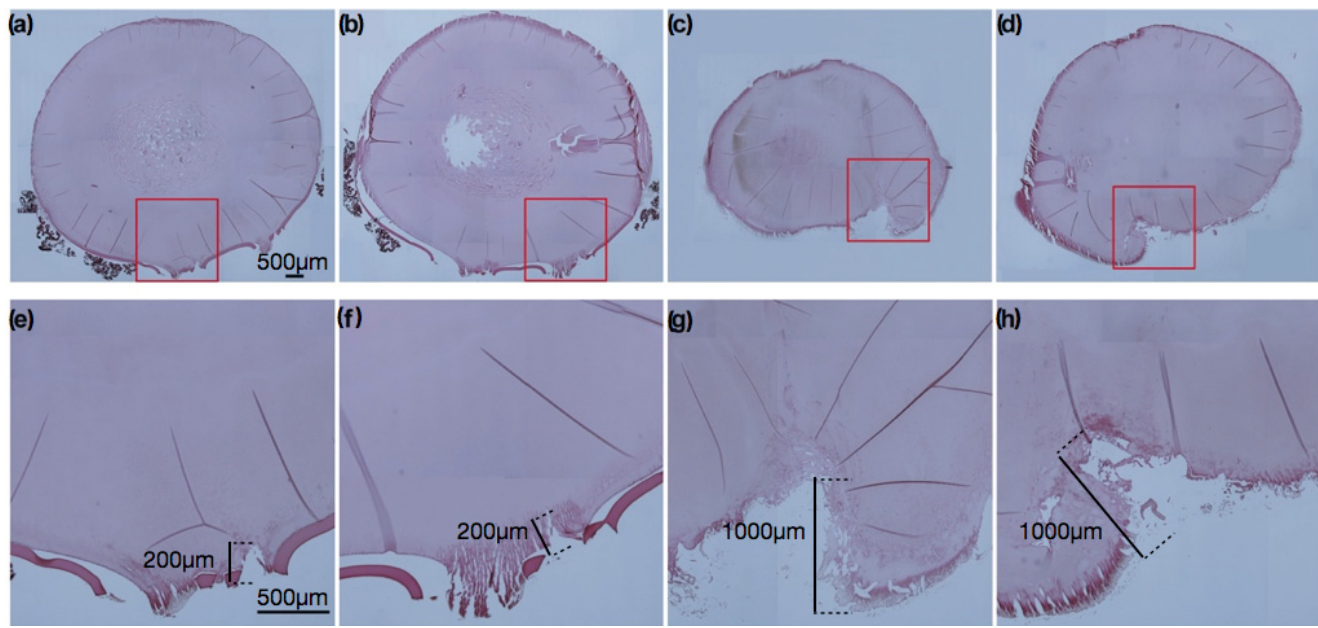

Fig. 7. Histological tissue slices of the laser-treated porcine lens. Panels (a), (b), (e), (f), are for SSTF focusing; panels (c), (d), (g), (h) are with a standard focus. The black scale bars shown in (a) and (e) are $500 \mu \mathrm{m}$, and the same scaling is used for all images within each row. Additional scale bars in (e)-(h) highlight the depth of laser modification in each case, $\sim 200$ um for (e), (f) and $\sim 1 \mathrm{~mm}$ for $(\mathrm{g})$, (h)

The panels left of center (Figs. 7(a), 7(b), 7(e), 7(f)) are ablated with the SSTF focus, while the panels right of center (Figs. 7(c), 7(d), 7(g), 7(h)) are ablated with a standard focus. The red boxes in the upper row of panels delineate the correspondingly magnified areas shown in the lower panels. From these images it is apparent that the damage only extends to $\sim 200$ micrometers for SSTF (panels (e) and (f)) whereas it extends to a millimeter with a standard focus (panels (g) and (h)). Additionally, the localization of the SSTF focal spot directly affects the integrity of the lens capsule, the darker red $\sim 10 \mu \mathrm{m}$ thick layer of tissue encapsulating the lens. In Figs. 7(e) and 7(f) the lens capsule remains intact outside of the ablated zone. Notice that various slices of capsule in the SSTF geometry can be seen, marking the rows from sequential passes, separated by $200 \mu \mathrm{m}$. Without SSTF, the lens capsule is absent, removed as a result of the bubble formation (Fig. 5) which extends significantly outside of the ablated zone.

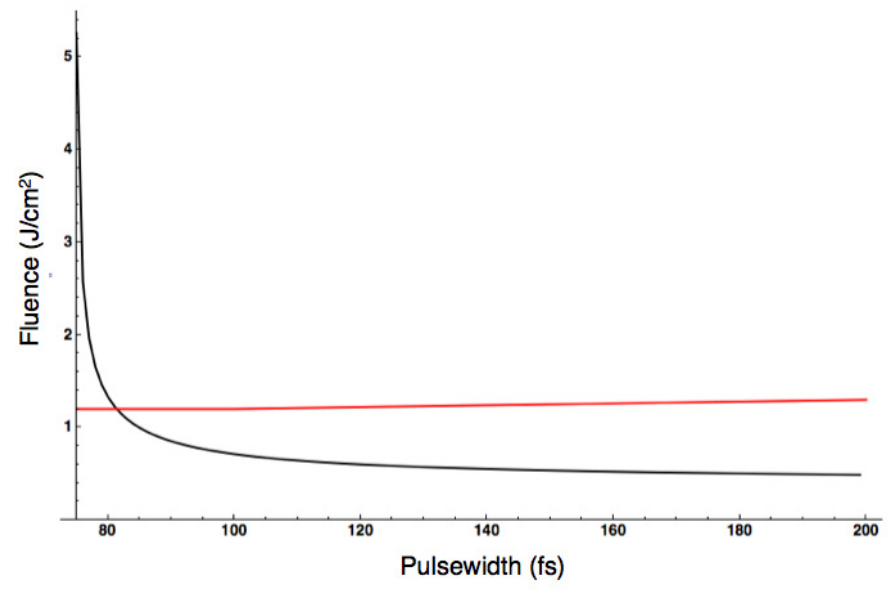

Fig. 8. Fluence as a function of laser pulse duration for the SSTF focus used in the eye ablation experiments. The black line is the fluence of the focus. The red line is the ablation threshold fluence as taken from reference [14]. There is a slight positive slope to the red ablation fluence line. 
What do we expect for a difference in the extent of the SSTF cuts relative to the standard focus? Figure 8 plots the SSTF fluence (in black) for the conditions used in these experiments versus pulse duration (which corresponds to axial position). The red line is the ablation threshold as measured by Giguère et al. [14]. For these short pulses, the measured ablation fluence has weak pulse width dependence and hence shows a slight positive slope. All points on the black curve that are above the red plot are above the ablation threshold. Thus for these focusing conditions the fluence is high enough to ablate out to a pulse duration of $\sim 83$ fs which corresponds to an axial distance of $\sim 160 \mu \mathrm{m}$ from the focal spot.

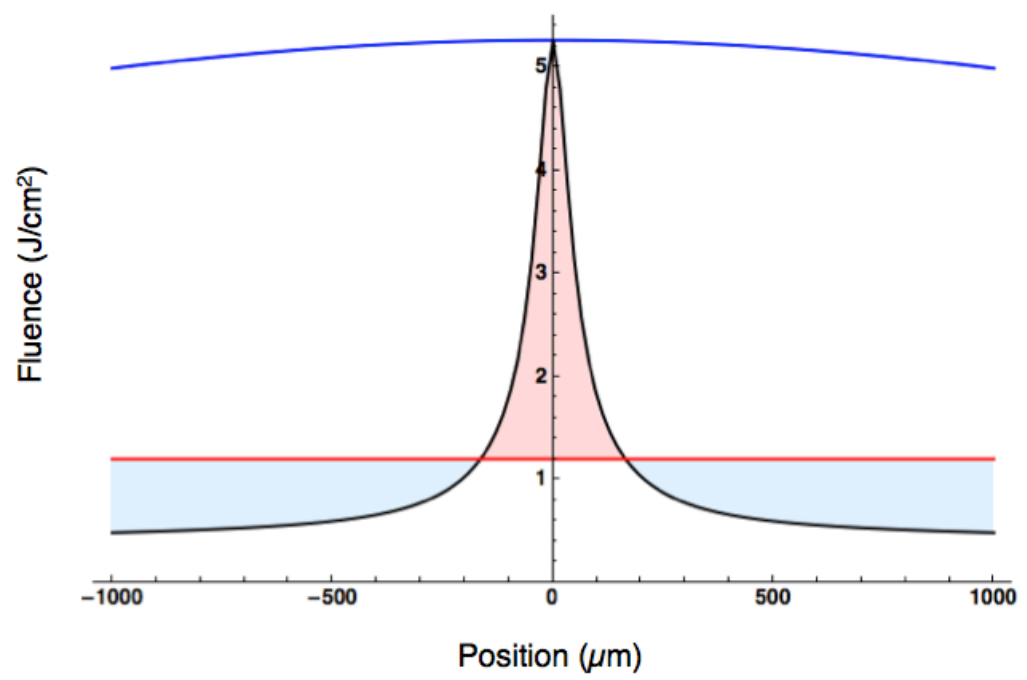

Fig. 9. Fluence as a function of axial position. Blue - is the fluence for the standard focus. Black - is the fluence for the SSTF focus. Red - is the measured ablation threshold (from [14]) for a $100 \mathrm{fs}$ pulse. The light red fill illustrates the portion of the SSTF focus that is above the ablation threshold and the region were damage could be expected. The light blue fill illustrates the region of the SSTF focus below the ablation threshold. Note that the standard focus (blue contour) is above the ablation threshold (red contour) for the entire region depicted in the plot.

Alternatively, the fluence profile of both the SSTF and standard focus can be plotted as a function of axial position, and compared relative to a single value of the ablation threshold $[14,15]$. This analysis is done in Fig. 9. The SSTF focus (black) is only above the ablation threshold (red) to a depth of $160 \mu \mathrm{m}$, while the standard focus (blue) is above the ablation threshold for millimeters. Figures 8 and 9 reinforce precisely what is seen in the histology. The extent of the SSTF ablation should be limited to several hundred micrometers, while the damage with the standard focus should extend to millimeters.

\#185757 - \$15.00 USD Received 21 Feb 2013; revised 11 Apr 2013; accepted 6 May 2013; published 8 May 2013 (C) 2013 OSA 1 June 2013 | Vol. 4, No. 6 | DOI:10.1364/BOE.4.000831 | BIOMEDICAL OPTICS EXPRESS 839 


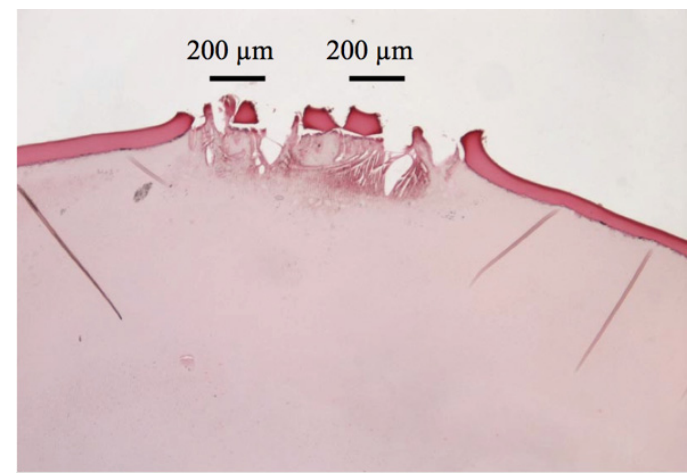

Fig. 10. Histological tissue slice of porcine lens. SSTF was employed and the sample was translated at a speed of $10.8 \mathrm{~mm} / \mathrm{s}$ through focus.

Finally, rapid ablation is also achievable with SSTF. Cutting at $\sim 58$ times above the ablation threshold at 18 times the translation speed $(10.8 \mathrm{~mm} / \mathrm{s})$ with a reduced beam aspect ratio $\left(\beta_{B A}\right.$ $=4)$ we see there is no compromise in precision when SSTF is implemented. As was done previously, the focus was placed at the surface of the lens, normally incident and four rows are cut. An image of the histology for the rapid ablation is shown in Fig. 10. In this instance, each spot of the tissue is only exposed to a single laser shot. The four individual rows can be seen.

\section{Conclusion}

To ablate materials or tissue cleanly, femtosecond lasers are typically operated at or slightly above the ablation threshold [1-5]. However, especially in regards to surgical implementations, as a result of variation in the tissue it is often desirable to ablate significantly above threshold to break up troublesome regions. The difficulty is that often the laser is focused to a moderate spot size $(\sim 10-15 \mu \mathrm{m})$ in order to ensure adequate working distances. The resultant low numerical aperture beams are susceptible to nonlinear effects such as self-focusing and the energy fluence cannot be raised aggressively above threshold without serious consequences. In contrast, we have shown here for the first time, that with SSTF even larger beam sizes (lower numerical aperture) can be accommodated and be operated well above threshold to help break up tissue, all the while maintaining controlled and predictable damage zones.

Finally it should be noted, that the SSTF approach does not require the short pulses used here. In fact, we show that there may be additional benefit to working with longer pulse durations. Many lasers in use in ophthalmic surgeries today have pulses durations on the order of $500 \mathrm{fs}$ (compared to the $75 \mathrm{fs}$ used here). SSTF can be implemented with these longer pulse duration systems; provided sufficient spectral dispersion can be imparted to the beam to reach the desired beam aspect ratio, the localization is independent of the pulse duration. The gains in these longer pulse systems maybe even more substantial since the ablation threshold at these pulses durations tends to exhibit a dependence that scales as the square root of the pulse duration, whereas for short pulses the energy fluence threshold for damage is only weakly dependent on pulse duration. 

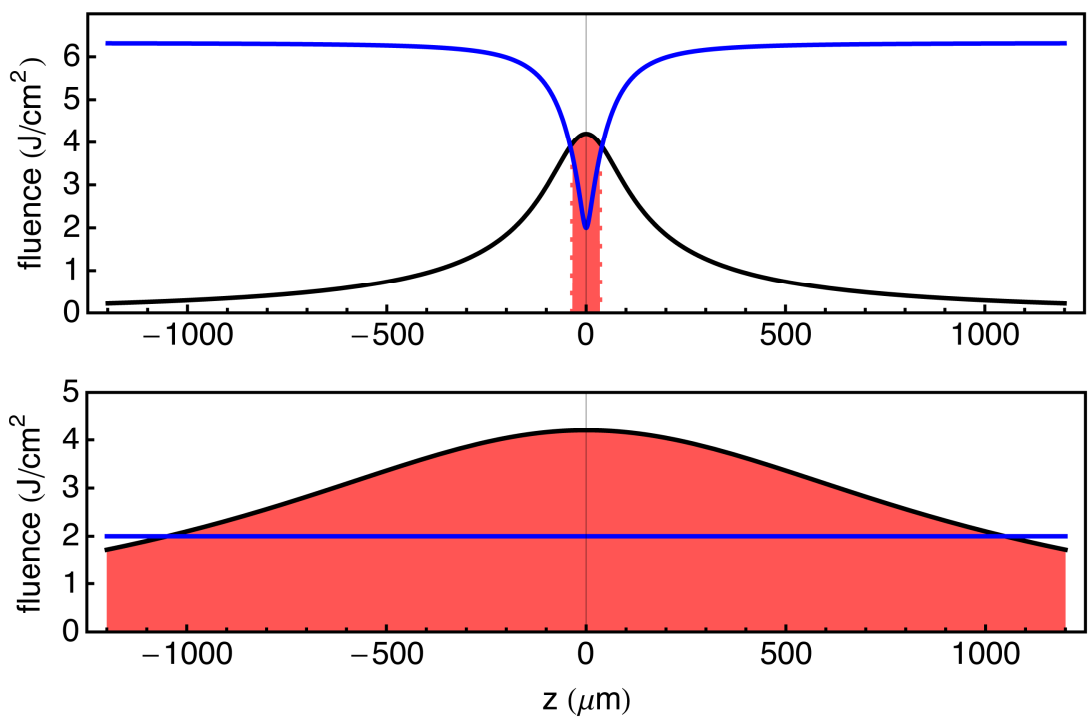

Fig. 11. Upper plot - SSTF fluence (black curve), blue is ablation threshold assuming a square root dependence on the ablation threshold. Red filling is region where the pulse fluence is above damage threshold. Lower plot - Standard focus fluence (black curve), blue is ablation threshold for a constant pulsewidth. Red filling is region where the pulse fluence is above damage threshold.

Figure 11 compares the gain in precision obtained for systems operating in this regime. The beam aspect ratio used for the SSTF calculation (Upper plot, Fig. 11) is 11. Note that the axial ablation zone extends more than 20 times further for the case of a standard focus compared to the SSTF focus.

\section{Acknowledgments}

This work was funded by the National Institute of Biomedical Imaging and Bioengineering under the Bioengineering Research Partnership EB-003832. C. D. and M. G. acknowledge support from the Air Force Office of Scientific Research under grants FA9550-10-1-0394 and FA9550-10-0561. 